\title{
Orbital effect and weak localization in the longitudinal magnetoresistance of Weyl semimetals NbP, NbAs, TaP, and TaAs
}

\author{
M. Naumann $\odot,{ }^{1,2}$ F. Arnold, ${ }^{1}$ M. D. Bachmann $\odot,{ }^{1}$ K. A. Modic, ${ }^{1}$ P. J. W. Moll, ${ }^{1}$ V. Süß $\beta,{ }^{1}$ M. Schmidt, ${ }^{1}$ and E. Hassinger $\oplus^{1,2}$ \\ ${ }^{1}$ Max Planck Institute for Chemical Physics of Solids, 01187 Dresden, Germany \\ ${ }^{2}$ Physik-Department, Technische Universität München, 85748 Garching, Germany
}

(Received 12 November 2019; revised manuscript received 6 January 2020; accepted 7 February 2020; published 10 March 2020)

\begin{abstract}
Weyl semimetals such as the TaAs family (TaAs, TaP, NbAs, NbP) host quasiparticle excitations resembling the long-sought-after Weyl fermions at special band-crossing points in the band structure denoted as Weyl nodes. They are predicted to exhibit a negative longitudinal magnetoresistance (LMR) due to the chiral anomaly if the Fermi energy is sufficiently close to the Weyl points. However, current jetting effects, i.e., current inhomogeneities caused by a strong, field-induced conductivity anisotropy in semimetals, have a similar experimental signature and therefore have hindered a determination of the intrinsic LMR in the TaAs family so far. This work investigates the longitudinal magnetoresistance of all four members of this family along the crystallographic $a$ and $c$ directions. Our samples are of similar quality as those previously studied in the literature and have a similar chemical potential, as indicated by matching quantum-oscillation frequencies. Care was taken to ensure homogeneous currents in all measurements. As opposed to previous studies where this was not done, we find a positive LMR that saturates in fields above $4 \mathrm{~T}$ in $\mathrm{TaP}, \mathrm{NbP}$, and $\mathrm{NbAs}$ for $B \| c$. Using Fermi-surface geometries from band-structure calculations that had been confirmed by experiment, we show that this is the behavior expected from a classical purely orbital effect, independent of the distance of the Weyl node to the Fermi energy. The TaAs family of compounds is the first to show such a simple LMR without apparent influences of scattering anisotropy. In configurations where the orbital effect is small, i.e., for $B \| a$ in NbAs and NbP, we find a nonmonotonous LMR, including regions of negative LMR. We discuss a weak antilocalization scenario as an alternative interpretation to the chiral anomaly for these results, since it can fully account for the overall field dependence.
\end{abstract}

DOI: 10.1103/PhysRevMaterials.4.034201

\section{INTRODUCTION}

Topological Weyl semimetals host three-dimensional linear band-crossing points of spin-polarized bands in their band structure, so-called Weyl nodes. At the nodes, the quasiparticle excitations behave like relativistic fermions, namely, chiral Weyl fermions [1]. Hence, these condensed-matter systems allow the study of effects which are otherwise accessible only in high-energy physics. In particular, our aim is to investigate the Adler-Bell-Jackiw anomaly or chiral anomaly, an imbalance of the number of left-handed and right-handed Weyl fermions in parallel electric and magnetic fields [2,3] in Weyl semimetals. There, the chiral anomaly contribution to the electrical conductivity should lead to a negative longitudinal magnetoresistance (LMR) [4,5]. Theoretically, its size decreases with increasing energy difference between the Fermi level and the Weyl node and with internode scattering, both present in real materials [5-7].

Published by the American Physical Society under the terms of the Creative Commons Attribution 4.0 International license. Further distribution of this work must maintain attribution to the author(s) and the published article's title, journal citation, and DOI. Open access publication funded by the Max Planck Society.
The prime Weyl semimetal candidate materials where the chiral anomaly might occur is the TaAs family, including $\mathrm{TaAs}, \mathrm{TaP}, \mathrm{NbAs}$, and $\mathrm{NbP}$. The presence of Weyl points in the band structure due to inversion symmetry breaking was predicted by Weng et al. [8] and Huang et al. [9]. All four materials have similar band structures that harbor Weyl nodes within $10-50 \mathrm{meV}$ from the Fermi energy. The calculated band structures have been confirmed by angleresolved photoemission spectroscopy (ARPES) and quantum oscillations (QO) [10-14]. Initial measurements of the LMR indeed revealed it to be negative, which was interpreted as evidence for the chiral anomaly (Refs. [15-18], and Ref. [19] and references therein).

However, there are two aims motivating a reinvestigation of the LMR in this family of compounds. First, it has been shown that applied magnetic fields induce a sizable conductivity anisotropy in these materials. This results in strong current inhomogeneities when field and current are aligned, so-called current jetting, which disguises the true, intrinsic, LMR [13,19-21]. In none of the above experiments was current jetting ruled out. Consequently, the first aim of this work was to detect the intrinsic $\rho_{\mathrm{zz}}$ in crystals of all members of this family by confirming current homogeneity during the measurement.

The second aim of this study was to interpret the results without solely looking for the chiral anomaly. There is a 
variety of other effects that can explain a negative LMR, such as weak localizations [22-24], magnetic impurities [25,26], a finite Berry curvature [6,27,28], higher-order corrections to the Boltzmann transport equations [29], or a negative Gaussian curvature [30] of the Fermi surface.

To achieve homogeneous currents and a reliable resistance measurement, we follow two routes: injecting the current in a homogeneous manner or increasing the aspect ratio by microstructuring techniques. The latter has been used before [31], and tiny indications of the chiral anomaly were detected on crystals where the chemical potential was near the Weyl node due to gallium implantation. In contrast, microstructured TaAs for $B \| c$ shows a positive and saturating LMR along the crystallographic $c$ direction [32]. This study focuses on samples of similar quality and chemical potential to those reported in the literature cited above (see Supplemental Material (SM) [33] Tables 1 and 2).

We find the intrinsic LMR to depend strongly on the crystallographic orientation of the current. The LMR along the $c$ direction rises and saturates for fields below $4 \mathrm{~T}$. The saturation value is in full agreement with the expected orbital MR based on the Fermi-surface geometry alone (Ref. [34] and SM chapter 1.6). Along the crystallographic $a$ direction the field dependence is weak and nonmonotonic. While in TaP orbital effects can again explain the LMR, in NbP and $\mathrm{NbAs}$ the field dependence is in agreement with weak-localization physics. The longitudinal transport in the TaAs family can therefore be explained without including any chiral or topological effects, suggesting that these effects are either absent or very small.

\section{EXPERIMENTAL METHODS}

Crystals of TaAs, TaP, NbAs, and $\mathrm{NbP}$ were grown by chemical vapor transport using iodine as transport agent. Details of the sample growth are described in the SM. The crystals showed residual resistivity ratios $[\mathrm{RRR}=$ $\mathrm{R}(300 \mathrm{~K}) / \mathrm{R}(2 \mathrm{~K})]$ of around 5-36 depending on sample and the orientation of current with respect to the crystallographic axis. Residual resistivities range from 1.2 to $7 \mu \Omega \mathrm{cm}$. These residual resistivities and RRRs are in the same range as what was reported in the literature, although they indicate a slightly elevated level of disorder compared to the best samples reported so far (see SM Table 1).

Most crystals of those materials depart from perfect compensation (equal number of electrons and holes) that is normally expected for semimetals, probably due to crystallographic imperfections below the detection limit of chemical analysis and beyond crystal growth control. Nevertheless, the chemical potential seems to be quite robust in samples produced in different labs, since many reports agree on quantum-oscillation frequencies in as-grown crystals (see SM Table 2). TaAs is the only confirmed compound in the family where mirror-symmetric pairs of Fermi-surface pockets with nonzero Berry flux through the individual Fermi surfaces are found, an assumed prerequisite for the chiral anomaly [12-14].

Analyzing the Shubnikov-de Haas oscillations in our crystals, we find similar levels of the chemical potential as reported in the literature $[13,14,35,36]$ (see SM Table 2). In general, QO frequencies $f$ are virtually identical to previous studies in both tantalum compounds $(\Delta f<1 \mathrm{~T})$, whereas they differ slightly for the $\mathrm{Nb}$ compounds $(\Delta f \approx 2 \mathrm{~T})$.

Because of the agreement of the size of the Fermi-surface pockets (given by quantum-oscillation frequencies), the lowtemperature charge-carrier densities only vary by a few percent compared to samples in previous reports. Therefore the residual resistivity changes are mainly caused by changes in the mobility and are a good indicator of sample quality.

The crystals were oriented in a Laue diffractometer, cut on a wire or dicing saw, and polished into a barlike shape. The resulting samples had dimensions of around 400-1000 $\mu \mathrm{m}$ in length, 200-400 $\mu \mathrm{m}$ in width, and about $100 \mu \mathrm{m}$ in thickness.

Current jetting primarily arises in materials with a large transverse magnetoresistance (MR). Compensated semimetals with a high mobility, such as bismuth or the TaAs family, have a transverse MR of around 1000 in magnetic fields of $10 \mathrm{~T}$ and are therefore predestined to exhibit this effect. If the current is injected into the sample through a point-shaped contact, the strong conductivity anisotropy will force it to progress as a jet along the field direction. This will lead to an apparent negative LMR, since the current is typically diverted away from the voltage contacts in an increasing magnetic field $[19,20]$.

For a homogeneous current injection, current contacts were soldered, covering the complete cross section of the samples. To get as local as possible information on the current distribution and homogeneity, up to three pairs of spot-welded voltage contacts distributed over the width of the samples (U1, U2, and U3) were employed. The contact geometry is depicted in Fig. 1(a).

In the literature, attempts had been made to solve the current jetting issue by using large current or voltage contacts made from silver paint. However, current contacts made from silver paint do not result in a homogeneous current injection [19]. Also, voltage contacts in the shape of a stripe of silver paint across the full width of the sample, as proposed by Ref. [17], do not detect the intrinsic LMR as sort of a current average (see SM Figs. 8 and 9).

Another possible source of current inhomogeneities are those caused by the sample inhomogeneity itself, such as stacking faults that were shown to exist in these materials $[18,37]$. However, since we interpret data only where several voltage contact pairs agree, this does not affect our results.

Focused ion beam (FIB) microstructured NbAs transport devices were fabricated using a gallium-based Helios NanoLab G3 CX machine. An overview of FIB-based device fabrication of quantum matter can be found elsewhere [38]. It has been shown, that microstructuring of TaAs-type Weyl semimetals using a FIB results in a tantalum- or niobium-rich surface layer [39]. This will become superconducting at temperatures between 2 and $4 \mathrm{~K}$, which is why the measurements on both $\mathrm{NbAs}$ microstructures were performed at $4 \mathrm{~K}$.

Since the conductivity anisotropy and hence current jetting effects are growing in increasing magnetic fields, all voltage contacts typically agree at low fields where they are less dependent on the current contact quality [see the black curves in Figs. 1(b) and 1(c)]. At higher fields, deviations between different contacts were visible in most cases. For qualitatively similar field dependencies with only small differences as in Figs. 1(b) and 1(c), the current distribution was 
(a)
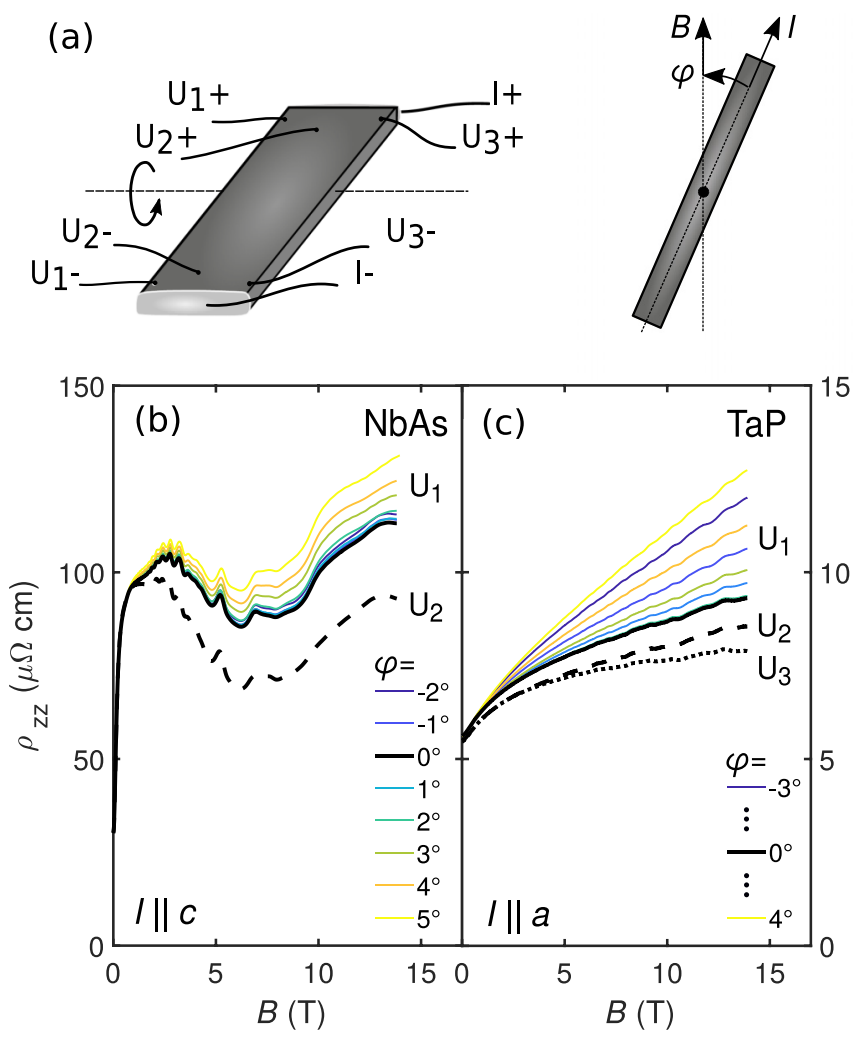

FIG. 1. (a) The employed contact geometry for bulk samples and the angle of rotation. (b) The longitudinal magnetoresistance in $\mathrm{NbAs}$ with current along $c$ for some angles around the $B \| I$ orientation. (c) A similar plot for TaP and current along $a$. Different line styles correspond to different contact pairs, and the black lines denote the orientation where $B \| I$. For better visibility, the angle dependence of only a single contact pair is shown.

deemed sufficiently homogeneous, and we will refer to the $\rho_{\mathrm{zz}}$ as "intrinsic".

It should be noted that even a perfectly homogeneous current injection is still susceptible to current jetting effects if the sample is not perfectly aligned in the external magnetic field. Under these circumstances, the "jet" will be as large as the sample cross section, passing through the sample in the direction of the misaligned magnetic field [20]. In order to spot this, field sweeps were performed at various angles $\varphi$ around the parallel orientation (see Fig. 1). The rotation around $\varphi$ was done in situ at $2 \mathrm{~K}$ in a 14-T Quantum Design (QD) physical-property measurement system (PPMS) using a QD rotator probe. Magnetic field sweeps were performed from -14 to $14 \mathrm{~T}$ (or vice versa), and data was subsequently symmetrized to exclude any Hall contribution to the signal. In these rotation studies, a clear sign of current jetting is when a contact showing a strong positive magnetoresistance in high fields would turn to a "negative resistance" upon rotating only a few degrees. The alignment around the second angle $\vartheta$ was done by eye under a microscope, leading to uncertainties of a few degrees.

For both $B \| a$ and $B \| c$, the symmetry of the system results in a conductivity tensor of reduced form with $\sigma_{i \mathrm{z}}=\sigma_{\mathrm{zi}}=0$ with $i=\mathrm{x}, \mathrm{y}$, where $\sigma_{\mathrm{zz}}$ can be easily retrieved by inverting $\rho_{\mathrm{zz}}$. In contrast, if the system is tilted slightly off a main crystallographic axis, the symmetries preserving this form are broken and $\sigma_{\mathrm{zz}}$ can only be extracted by a full tensor inversion of $\hat{\rho}$ and vice versa. This leads mainly to an admixture of the transverse magnetoconductivity to the signal that smoothly grows with the angle. Both niobium compounds with current along $a$ had an increasing resistance in high fields with this angle dependence. In that case, only the low-field behavior, where the transverse MR goes to zero, was ascribed to the LMR.

For reasons of clarity, the main text contains mainly results from one voltage contact pair on each sample. The reader is referred to the SM, where the full results are shown.

\section{POSSIBLE PHENOMENA CAUSING A MAGNETORESISTANCE}

In order to aid the interpretation of the data shown hereafter, we would like to give an overview over effects contributing to an LMR.

(1) The orbital LMR of a free electron gas with spherical Fermi surface is known to be zero [21]. However, a particular corrugation of the Fermi surface gives rise to a finite LMR caused by orbital effects [20,34]. The model detailed in Ref. [34] is based on a Boltzmann approach to the transport problem and takes only the geometry of the Fermi surface into account. The scattering time is isotropic. In a nutshell, if the average Fermi velocity component in the direction of field and current varies along a cyclotron orbit, there will be a finite LMR and the expected change in conductivity in the high-field limit $\delta \sigma / \sigma_{0}=[\sigma(B=\infty)-\sigma(0)] / \sigma(0)$ can be obtained. Here we calculate $\delta \sigma / \sigma_{0}$ of all compounds and in both crystallographic current or field directions using the Fermi surfaces from DFT results that showed good agreement with quantum oscillations [12-14]. Results are presented in Table 3 in the SM. How fast the saturation value is reached depends on the mobility $\mu$, and the orbital magnetoresistance is given by

$$
\sigma_{\mathrm{orb}}(B)=\frac{n e \mu}{1+(\mu B)^{2}},
$$

with the charge-carrier density $n$. Since this is a decreasing function in $B$, the magnetoresistance is positive. Despite this, there is a recent theoretical prediction that a negative Gaussian curvature of the Fermi surface can cause a negative LMR [30].

(2) The chiral anomaly contributes a positive term to the total longitudinal conductivity when magnetic and electric fields are parallel [4,5]. In the semiclassical regime,

$$
\sigma_{\mathrm{CA}}=C B^{2}
$$

with $C$ being a constant $[5,6]$.

In the simplest possible model, the size of this contribution $C$ decreases with the distance of the Fermi energy to the Weyl nodes by $\left|E_{\text {Weyl }}-E_{\mathrm{F}}\right|^{-2}$ [5], but the exponent decreases to at least -3 in a more realistic model [7]. In this model, a distance of more than $5 \mathrm{meV}$ is sufficient to make the chiral conductivity contribution negligible. Note that calculations for the real band structures are missing, implying absolute numbers to be different in real materials.

(3) Electron self-interference effects, also called weak (anti-) localization, can induce both positive and negative MR. 
These effects occur when the electron dephasing time $\tau_{\phi}$ is larger than the scattering time $\tau$. Whether a weak localization or a weak antilocalization occurs depends on the symmetry of the system [40]. In the materials investigated here, the existence of strong spin-orbit coupling (leading to spin-polarized Fermi surfaces and broken spin-rotational symmetry) puts them in the symplectic symmetry class [40] and therefore only a weak antilocalization (WAL) is applicable. Note that in a two-dimensional (2D) or in a Weyl system, a WAL will appear as a purely positive MR $[40,41]$. In contrast, in topologically trivial three-dimensional (3D) systems, there is a competition of singlet and triplet transport contributions governed either by the electron dephasing time alone (singlet) or a mixture of dephasing and spin-orbit scattering time [triplet, see Eq. (4)] [22-24]. This can result in a nonmonotonous field dependence. In the literature, this competition is often referred to as a crossover between an antilocalization and a localization, with the described situation in a 2D system in mind [18].

The additional conductivity contribution is different for Weyl systems, $\sigma_{\mathrm{WWAL}}(B)$ [41], and those with a "trivial," nonchiral electronic structure, $\delta \sigma_{\mathrm{WAL}}(B)$ [22-24]. The magnetic field dependence of the conductivity of a Weyl weak antilocalization (WWAL) can be expressed as

$$
\begin{aligned}
\delta \sigma_{\mathrm{WWAL}}(B) & =\sigma_{\mathrm{WWAL}}(B)-\sigma_{\mathrm{WWAL}}(0) \\
\sigma_{\mathrm{WWAL}}(B) & =\frac{2 e^{2}}{(2 \pi)^{2} h} \int_{0}^{1 / l} d x\left[\psi_{l_{B}}(l)-\psi_{l_{B}}\left(l_{\phi}\right)\right] \\
\psi_{l_{B}}(z) & =\Psi\left(\frac{l_{\mathrm{B}}^{2}}{z^{2}}+l_{\mathrm{B}}^{2} x^{2}+\frac{1}{2}\right) .
\end{aligned}
$$

Here, $l$ denotes the mean free path, $l_{\phi}$ the dephasing length, $l_{\mathrm{B}}=\sqrt{\hbar /(4 e B)}$ the magnetic length, and $\Psi$ the digamma function [41].

The correction to the conductivity due to a "trivial" weak antilocalization in 3D is given by

$$
\begin{gathered}
\sigma(B)=\sigma(0)+\delta \sigma_{\mathrm{WAL}}(B) \\
\delta \sigma_{\mathrm{WAL}}(B)=-\frac{e^{2}}{2 \pi^{2} \hbar} \sqrt{\frac{e B}{\hbar}} \\
\times\left[\frac{1}{2} f_{3}\left(\frac{B}{B_{\phi}}\right)-\frac{3}{2} f_{3}\left(\frac{B}{B_{2}}\right)\right] \\
B_{2}=B_{\phi}+\frac{4}{3} B_{s o}, \\
f_{3}(x)=\sum_{n=0}^{\infty}\left[2(n+1+1 / x)^{1 / 2}\right. \\
\left.-2(n+1 / x)^{1 / 2}-(n+1 / 2+1 / x)^{-1 / 2}\right] .
\end{gathered}
$$

The quantities $B_{\phi, s o}$ are parameters which are determined by the dephasing or spin-orbit scattering times via the relation $B_{i}=\hbar /\left(4 e D \tau_{i}\right)$ with $i=(\phi$, so $)$ and $D$ being the diffusion constant. Apparently, the WWAL will result in a negative conductivity contribution, i.e., a positive magnetoresistance. In contrast, the "trivial" 3D WAL will result in a negative magnetoresistance at high fields, which may be positive at low fields depending on the particular choice of the spin-orbit scattering time $\tau_{\text {so }}$ relative to the dephasing time $\tau_{\phi}$ [22-24].

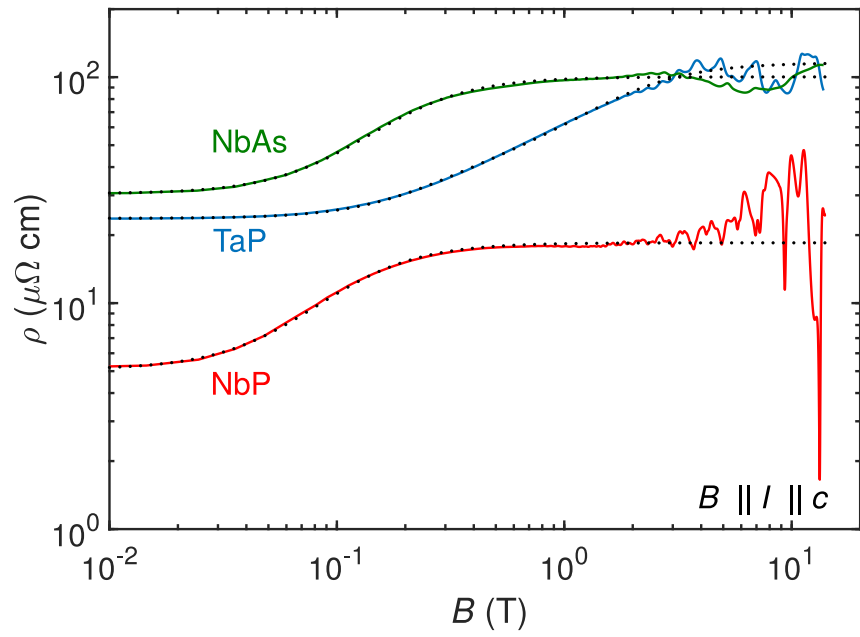

FIG. 2. The longitudinal magnetoresistance for TaP, NbAs, and $\mathrm{NbP}$ with current along $c$. The fits to the orbital model are shown as a black dotted line.

It should be noted that these models are the full results of the theory. WAL types of effects have already been discussed in conjunction with a chiral anomaly in both arsenides $[15,17,18]$ based on approximations such as, e.g., $\rho \propto \sqrt{B}$, which is only true for a rather large separation of $l$ and $l_{\phi}$.

(4) Magnetic impurities can lead to a negative magnetoresistance, known from, e.g., Kondo systems. Upon alignment of the magnetic moments by the external magnetic field, the disorder is decreased and so is the resistance [25]. We found an upper threshold of 100 ppm magnetic impurities in our samples based on magnetization results (see Sec. 1.5 in the SM). Traditional systems with similar impurity concentrations, such as AuFe, host a sizable Kondo effect [26]. However, the bandwidth and density of states at the Fermi energy in semimetals is appreciably lower. Since the Kondo temperature depends linearly on the former and exponentially on the latter, it should be suppressed by several orders of magnitude. Therefore we rule out that magnetic impurities influence the LMR in this study.

(5) Other reasons for a longitudinal MR are anisotropic scattering, macroscopic inhomogeneities, barrier inhomogeneities in superlattices, or a modification of the density of states in field (see Ref. [34] and the references therein), but these will not be discussed here. The same applies to secondorder band-structure effects, including the Berry curvature [28]. As shown below, the effects that are enumerated above are sufficient to describe the experimental results.

From this list, it can be seen that a negative LMR may be caused by a chiral anomaly, a weak antilocalization, magnetic impurities, or band-structure effects such as a negative Gaussian curvature. Therefore, the implication used occasionally that a negative LMR proves a material to be topologically nontrivial is wrong.

\section{IV. $c$-AXIS TRANSPORT}

Figure 2 shows the longitudinal magnetoresistance of TaP, $\mathrm{NbP}$, and $\mathrm{NbAs}$ for current along the crystallographic $c$ direc- 
tion. In all three compounds, the resistivity rises and saturates at fields between 2 and $4 \mathrm{~T}$. In TaP, higher fields are necessary to reach the saturation than in the niobium compounds. All signals are superimposed by quantum oscillations; in $\mathrm{NbP}$ they are extremely pronounced, and their size is of the same order as the rise in resistivity in field. In this paper, we concentrate on the background MR. Since all contact pairs agree qualitatively in the measurements shown here, this is the intrinsic LMR (see SM, Figs. 1, 6, and 10). This is also supported by the rather small angular dependence of the initial increase.

The TaAs crystals at our disposal were too small along the $c$ axis to make samples suitable for meaningful measurements.

There are two possible effects contributing to a positive LMR: a WAL and orbital effects. Since the inherent negative LMR of the WAL is clearly absent, only orbital effects can be at the origin of the increasing resistivity.

The fit presented as a dotted line in Fig. 2 takes two orbital contributions (from warped parts of the Fermi surface of the electron and the hole pocket) and a field-independent conductivity channel $\sigma_{0}$ (from unwarped parts of the FS) into account:

$$
\begin{gathered}
\sigma(B)=\sigma_{\infty}+\sigma_{\mathrm{orb} 1}\left(B, \mu_{1}, n_{1}\right)+\sigma_{\mathrm{orb} 2}\left(B, \mu_{2}, n_{2}\right), \\
\rho(B)=\sigma(B)^{-1} .
\end{gathered}
$$

In this model, $\mu_{1 / 2}$ and $n_{1 / 2}$ are the electron/hole mobility and carrier densities and were treated as free parameters. In both $\mathrm{Nb}$ compounds, only one orbital term is needed, which is why $n_{2}$ was set equal to zero: in $\mathrm{NbAs}$ one band dominates the $c$-axis transport (see SM Table 3), and in $\mathrm{NbP}$ both contributing bands have similar mobilities, making them indistinguishable [42]. In the $\mathrm{Nb}$ compounds, the fits were performed between 0 and $4 \mathrm{~T}$, in TaP between 0 and $6 \mathrm{~T}$, since the resistivity saturates at higher fields. The cutoff was introduced to avoid any influence of the pronounced quantum oscillations and to keep more weight on the low-field increase, which was also the reason why it was performed on log-scaled data. The resulting parameters can be found in SM Table 5. The relative change of conductivity in field $\delta \sigma / \sigma_{0, \exp }$ is well in accordance with the expected one from the DFT Fermi surface alone, $\delta \sigma / \sigma_{0 \text {,th }}$ [34] (see SM Table 3). Also, the mobilities agree well with literature values of the $a$-axis mobilities $\mu^{a}$, as determined from a zero-field Drude model or two-band Hall fits [12-14,36]. The relatively simple model by Pal and Maslov describing semiclassical orbital effects of an irregular Fermi surface with isotropic scattering times seems to capture the essential physics of the LMR in NbAs, NbP for $B \| c$ and in both field directions in TaP. Small-angle scattering must also be negligible, since it increases the MR [43]. This result also implies that the chiral anomaly contribution is either absent or very small, in contradiction to previously published reports [18]. Note that the energy distance of the Weyl node to the Fermi level $\left|E_{\text {Weyl }}-E_{\mathrm{F}}\right|$ from the DFT band structures is smaller in NbAs $(5 \pm 5 \mathrm{meV})$ than in $\mathrm{NbP}(10 \pm 5 \mathrm{meV})$, $\mathrm{TaP}(13 \mathrm{meV})$, and TaAs (13 meV) [12-14]. However, there appears to be no correlation between $\left|E_{\mathrm{Weyl}}-E_{\mathrm{F}}\right|$ and the occurrence or size of a negative LMR.

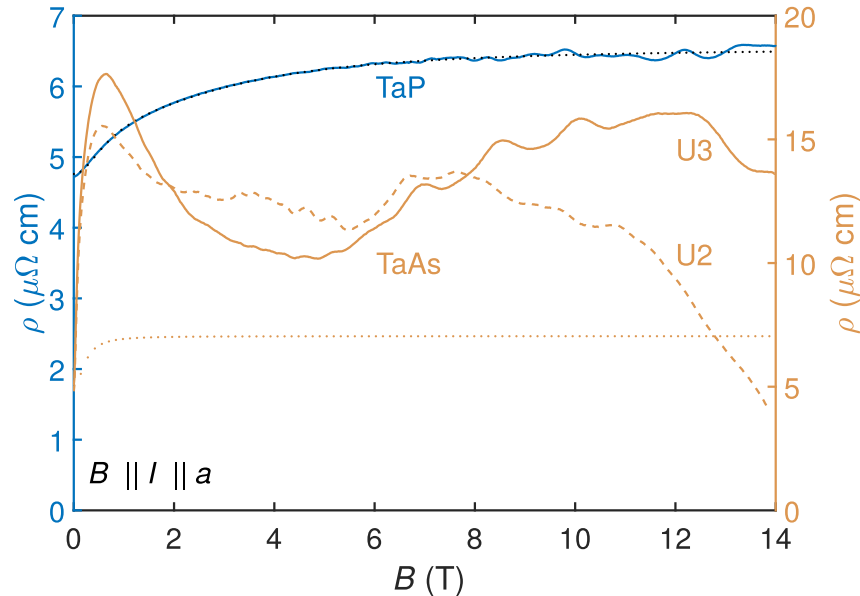

FIG. 3. The longitudinal magnetoresistance for both tantalum compounds with current along $a$. The lower orange dotted line indicates the expected orbital LMR in TaAs with a hypothetical 5 $\mathrm{T}^{-1}$ mobility as an illustration.

\section{V. $a$-AXIS TRANSPORT}

The $a$-axis transport in TaP (Fig. 3) strongly resembles the $c$-axis transport. No sign of any negative magnetoresistance is present. Here, all contact pairs agreed rather well (see SM, Figs. 2 and 3). Therefore the resistance curve shown reflects the true longitudinal magnetoresistance in TaP. A second sample gave the same qualitative field dependence (SM Fig. 2). The expected conductivity change for the $a$-axis transport in TaP (see Fig. 3) based on the FS geometry is also in agreement with the experimental results. Fit results are presented in the SM, Table 5. Again, the high-field saturation of the magnetoresistance agrees with the expectations based on the Fermi-surface corrugation.

As expected for this field orientation, the mobilities are lower. Due to the asymmetry of the Fermi surface, the effective masses are a factor of about 7 higher, while the mobility anisotropy we find is about 3 .

For TaAs, both voltage contacts are shown in Fig. 3. Given the different field dependence of the resistivity curves, we deem the current homogeneity insufficient to make reliable statements regarding the intrinsic LMR. However, both curves concordantly show a hump at low field followed by a saturation at high fields, likely intrinsic features of the MR. It resembles the results shown in [32], where $c$-axis transport data is presented. The initial increase, though, is beyond what is expected from the orbital contribution, as can be seen by comparing the data and the estimated orbital background (dotted line) in Fig. 3. Strong small-angle scattering can enhance the longitudinal MR [43]. However, the similarity of the hump in TaAs with that in NbAs hints to weak-antilocalization physics, as discussed in the next paragraph.

In NbP, Fig. 4, we can see the magnetoresistance to be rather small, only of the order of $25 \%$ in total. The signal decreases at low fields and increases almost linearly afterwards. Since the downturn at low fields shows up the same way in all three contact pairs (see SM, Fig. 12), we believe this to be of intrinsic origin. In higher fields, signs of current jetting appear, such as disagreement between contact 


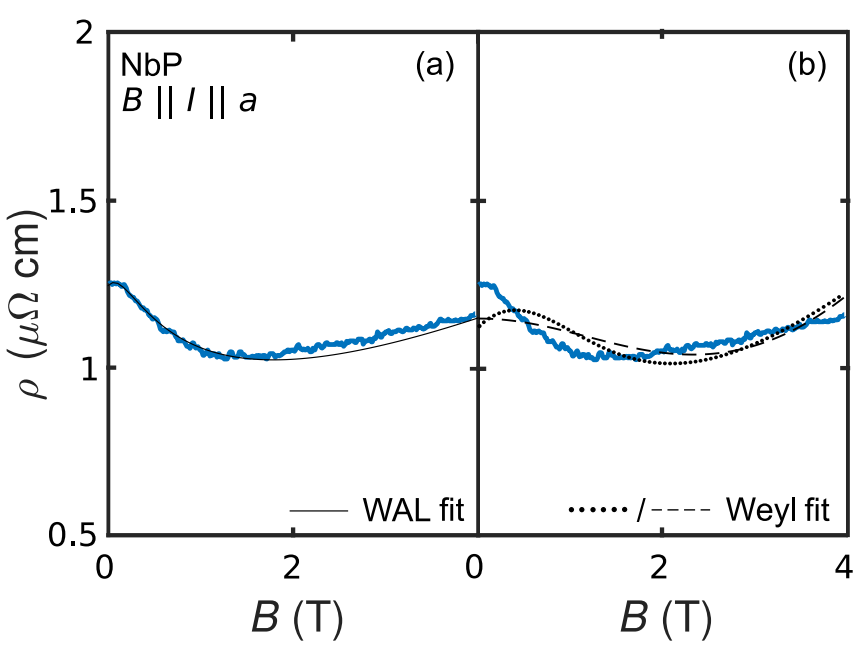

FIG. 4. The longitudinal magnetoresistance of $\mathrm{NbP}$ with current along $a$. The overall changes in resistivity are rather small. The black lines are fits according to (a) the WAL model (solid line), Eq. (7), and (b) the Weyl model (dotted: linear $f_{\mathrm{BG}}$, dashed: quadratic $f_{\mathrm{BG}}$ ), Eq. (8), with the Weyl WAL contribution constricted to zero.

pairs and negative voltages in the angle dependence (see SM Figs. 12 and 13). For the following interpretation the focus is on the low-field behavior, whereas the high-field increase is attributed to a misalignment of the sample in field.

The overall changes in resistivity are slightly higher in $\mathrm{NbAs}$, as shown in Fig. 5. At the lowest fields there is an increase in resistivity, while the signal beyond $0.3 \mathrm{~T}$ resembles that of $\mathrm{NbP}$. We also attribute the high-field resistivity increase to a misalignment of the sample's main axis with respect to the magnetic field. Although we had only one working voltage contact on this FIB microstructure, there are a number of reasons why we consider the signal to be reliable at low field. Measurements on a second microstructured sample with $I \| c$ present no difference between the different voltage contacts

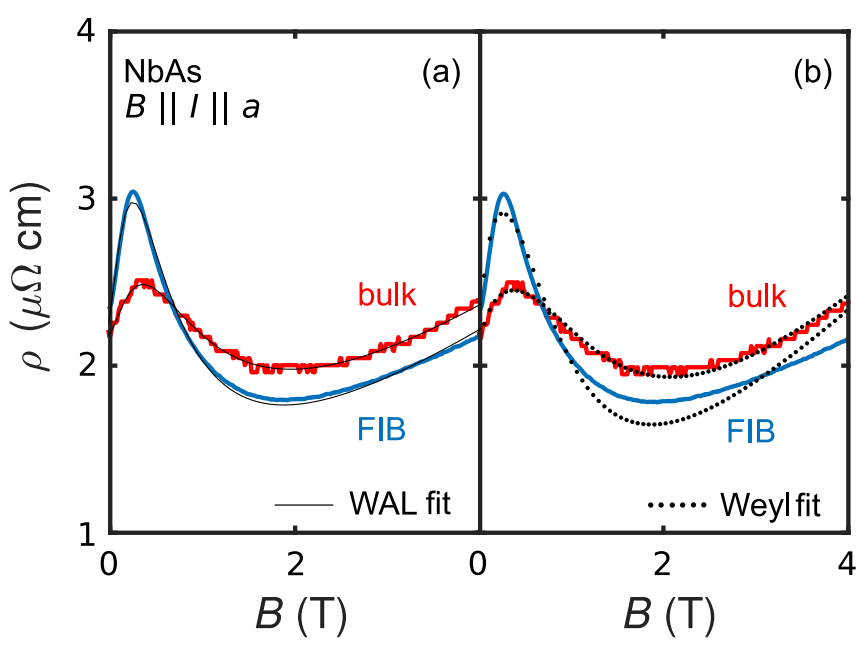

FIG. 5. The longitudinal magnetoresistance of both a bulk (red) and a microstructured sample (blue) of NbAs. The black lines denote fits according to (a) to the weak antilocalization model (solid), Eq. (7), and (b) according to the "Weyl model" (dashed line), Eq. (8). up to about $1 \mathrm{~T}$ and an almost field-independent difference of about $20 \%$ at fields greater $4 \mathrm{~T}$ (see Fig. 1(b)). Current jetting effects are suppressed once the aspect ratio is of the order of the square root of the conductivity anisotropy ratio $\sqrt{\sigma_{\|} / \sigma_{\perp}}$ [20]. We estimate this using resistivities to be $\sqrt{\rho_{B\|a, I\| c} / \rho_{B \| I|| a}}=7$ at $0.5 \mathrm{~T}$ and 20 at $4 \mathrm{~T}$. Since the microstructure has dimensions of $2.75 \mu \mathrm{m} \times 4.83 \mu \mathrm{m} \times 88 \mu \mathrm{m}$ $(\mathrm{w} \times \mathrm{t} \times 1)$, the samples' aspect ratio of about 18 is close to the conductivity anisotropy ratio.

The fact that the overall $a$-axis LMR in both $\mathrm{Nb}$ compounds is smaller than the $c$-axis one is in perfect agreement with the expected orbital MR based on the FS shape, which suggests a tiny $\delta \sigma / \sigma_{0}=0.075$ in $\mathrm{NbAs}$ and 0.097 in $\mathrm{NbP}$ (see SM, Table 3). As a consequence, we neglect the orbital effect in the following analysis. Both materials show a strong field dependence in low fields, including regions of negative magnetoresistance. In the following, we therefore discuss two possible scenarios for the nonmonotonic ( $\mathrm{NbAs}$ ) or negative $(\mathrm{NbP})$ behavior in low magnetic fields. In the "WAL" scenario, a (topologically) trivial 3D weak antilocalization $\delta \sigma_{\mathrm{WAL}}$ describes the full low-field behavior:

$$
\rho_{\mathrm{WAL}}(B)=\left[\sigma_{0}+A \delta \sigma_{\mathrm{WAL}}\left(B_{\phi}, B_{\mathrm{so}}\right)\right]^{-1}+f_{\mathrm{bg}}(B) .
$$

In the "Weyl" scenario, Weyl fermions dominate the LMR, causing a negative WWAL correction to the conductivity $\delta \sigma_{\mathrm{WWAL}}$ and at higher fields a positive chiral anomaly contribution $\sigma_{\mathrm{CA}}$ :

$$
\rho_{\mathrm{Weyl}}(B)=\left[\sigma_{0}+A \delta \sigma_{\mathrm{WWAL}}\left(l, l_{\phi}\right)+\sigma_{\mathrm{CA}}\right]^{-1}+f_{\mathrm{bg}}(B) .
$$

Both models include a constant conductivity contribution $\sigma_{0}$ and a function $f_{\mathrm{bg}}(B)$ accounting for the slowly increasing resistivity background at higher fields assumed to be stemming from a small misalignment. A simple linear function $f_{\mathrm{bg}}(B)=$ $d B$ was used as the simplest possible function fulfilling the requirement of $f_{\mathrm{bg}}(B \rightarrow 0) \rightarrow 0$ ( $c$ is a constant). Since this caused a hump at low fields in the fit, a quadratic background was also tried in NbP [see dotted (linear) vs dashed (quadratic) lines in Fig. 4]. In NbAs, this functional difference did not change the fit parameters significantly.

Results of these fits can be seen as black dotted lines in Figs. 4 and 5. The resulting fit parameters are given in full in the Supplemental Material, whereas we discuss only the crucial ones here.

The fit according to the weak antilocalization scenario describes the low-field behavior of both $\mathrm{NbP}$ and $\mathrm{NbAs}$ very well. We now check if the model is applicable at all. This is done by verifying the hierarchy of timescales $\tau \ll \tau_{\text {so }} \ll \tau_{\phi}$, with the electron dephasing time $\tau_{\phi}$, spin-orbit scattering time $\tau_{\text {so }}$, and total scattering time $\tau$. From the fit parameters $B_{\phi}=$ $9.7 \mathrm{mT}\left(17 \mathrm{mT}\right.$ ) and $B_{\mathrm{so}}=72 \mathrm{mT}$ (or $23 \mathrm{mT}$ ) for $\mathrm{NbAs}$ (or $\mathrm{NbP}$ ) we extract the dephasing and scattering times $\tau_{\phi}$ and $\tau_{\text {so }}$ by using an estimation of the diffusion constants $D$ (for details of the extraction see the SM, 1.9.1). For NbAs we find the inequality $\tau \ll \tau_{\text {so }} \ll \tau_{\phi}$ is fulfilled for the electron band such as $0.5<3<20 \mathrm{ps}$, whereas in $\mathrm{NbP}$ it barely is $1.0<5<8$ ps. It should be kept in mind that scattering times are typically associated with uncertainties up to 1 order of magnitude. 
With this large error bar in mind, we can state that the necessary hierarchy for a WAL to occur $\left(\tau \ll \tau_{\text {so }} \ll \tau_{\phi}\right)$ is mainly fulfilled. The size of the MR we observe is large compared to other reports of weak-localization MR [22,24]. Since this study is intended to investigate the field dependence of the LMR, a detailed temperature dependence that would be needed to confirm the WAL scenario is beyond the scope of this work. An open question is also why the WAL appears only in the $a$-axis transport and not in the $c$ axis.

In Figs. 4(b) and 5(b), a fit to the LMR in the Weyl scenario is shown. In NbP, the Weyl fit is performed without the WWAL contribution, since there is no low-field increase observable. This model cannot describe the experimental data, both with a linear and quadratic $f_{\mathrm{BG}}$, as shown as dotted (linear) and dashed (quadratic) lines in Fig. 4. The agreement is better in $\mathrm{NbAs}$, although it is clearly worse compared to the WAL model (Fig. 5). The fit parameters (Table 6 in the SM) indicate that the mean free path is of the same size as the dephasing length $l \simeq l_{\phi}$ and consequently, that the necessary separation of the two length scales is absent.

Our results presented here therefore reveal a number of observations which cannot be reconciled with the interpretation of a chiral anomaly being responsible for the negative LMR observed in these materials for $B \| a$ :

(1) In NbAs, the Weyl WAL fit of the upturn in resistivity at low fields yields unphysical fit parameters. But other possible origins of the upturn in the Weyl picture are absent since the orbital contribution is too small. Also, the presence of a trivial WAL responsible for the positive LMR would imply the presence of a negative LMR as well. The presence of two effects causing a negative LMR would reduce the individual contribution of the chiral anomaly to a rather low level and make its distinction and study even more cumbersome.

(2) In NbP no good agreement between the Weyl model and the data could be achieved.

(3) The possible influence of the negative Gaussian curvature of the Fermi surfaces has not been investigated or discussed so far. The "banana"-shaped Fermi surfaces present in all materials of this family have large regions of negative Gaussian curvature (positive curvature in one direction and negative in the perpendicular direction), which might very well contribute to the negative LMR [30].

Given that the relatively simple picture of a weak antilocalization is in agreement with the low-field data of both $\mathrm{NbAs}$ and $\mathrm{NbP}$, we assume this to be the likely cause of their LMR for $B \| a$.

\section{CONCLUSION}

The aim of this study was the achievement of a homogeneous current distribution in the measurement of the longitudinal magnetoresistance of the TaAs family of Weyl metals. We achieved this in all materials but TaAs and found especially the $c$-axis transport to exhibit a strictly positive LMR. While the $a$-axis transport in TaP strongly resembled the $c$-axis counterpart, both niobium compounds showed a negative or even nonmonotonic behavior.

Our second intention was to interpret the data without the strong expectation to find the chiral anomaly. Indeed, we found a number of possible effects which may exhibit a negative LMR and which have not been ruled out so far in the literature.

We found the $c$-axis transport in all compounds to be dominated by an orbital magnetoresistance. This is in full agreement with calculations of the expected size of the orbital LMR based on the Fermi-surface corrugation and the fit results to the LMR.

In contrast, the $a$-axis transport in both niobium compounds is more likely explained by a WAL model than a chiral anomaly. Given the qualitative similarity of the $a$-axis transport in TaAs and the excess of positive LMR found here, we believe it to be crucial to rule out the WAL scenario before any claims regarding the observation of a chiral anomaly can be made.

To our knowledge, a convincing evidence for the chiral anomaly, such as the expected increase of chiral currents when the chemical potential is shifted towards the Weyl nodes, is still missing.

\section{ACKNOWLEDGMENTS}

The authors would like to acknowledge the Max-Planck Society for their support of the MPRG, "Physics of Unconventional Metals and Superconductors." E.H. and M.N. acknowledge support from the German Research Foundation through the project TRR80, "From Electronic Correlations to Functionality" (Project No. 107745057). Also, we would like to thank Elio König-Tarasevich, Tobias Meng, Kamran Behnia, Yuki Fuseya, Thilo Klopp, Patrick Seiler, and Andy Mackenzie for stimulating and fruitful discussions.
[1] H. Weyl, Elektron und Gravitation. I, Zeitschrift Physik 56, 330 (1929).

[2] S. L. Adler, Axial-vector vertex in spinor electrodynamics, Phys. Rev. 177, 2426 (1969).

[3] J. Bell and R. Jackiw, A PCAC puzzle: $\pi_{0} \rightarrow \gamma \gamma$ in the $\sigma$ model, Nuov. Cim. A 60, 47 (1969).

[4] H. B. Nielsen and M. Ninomiya, The Adler-Bell-Jackiw anomaly and Weyl fermions in a crystal, Phys. Lett. B 130, 389 (1983).

[5] D. T. Son and B. Z. Spivak, Chiral anomaly and classical negative magnetoresistance of Weyl metals, Phys. Rev. B 88, 104412 (2013).
[6] P. Goswami, J. H. Pixley, and S. Das Sarma, Axial anomaly and longitudinal magnetoresistance of a generic three-dimensional metal, Phys. Rev. B 92, 075205 (2015).

[7] A. Johansson, J. Henk, and I. Mertig, Chiral anomaly in type-I Weyl semimetals: Comprehensive analysis within a semiclassical Fermi surface harmonics approach, Phys. Rev. B 99, 075114 (2019)

[8] H. Weng, C. Fang, Z. Fang, B. A. Bernevig, and Xi Dai, Weyl Semimetal Phase in Noncentrosymmetric TransitionMetal Monophosphides, Phys. Rev. X 5, 011029 (2015).

[9] S.-M. Huang, S. Xu, I. Belopolski, C. Lee, G. Chang, B. Wang, N. Alidoust, G. Bian, M. Neupane, C. Zhang et al., A Weyl 
Fermion semimetal with surface Fermi arcs in the transition metal monopnictide TaAs class, Nat. Commun. 6, 7373 (2015).

[10] Z. K. Liu, L. X. Yang, Y. Sun, T. Zhang, H. Peng, H. F. Yang, C. Chen, Y. Zhang, Y. F. Gao, D. Prabhakaran, M. Schmidt, Z. Hussain, S.-K. Mo, C. Felser, B. Yan, and Y. L. Chen, Evolution of the Fermi surface of Weyl semimetals in the transition metal pnictide family, Nat. Mater. 15, 27 (2015).

[11] S.-Y. Xu, I. Belopolski, N. Alidoust, N. Neupane, G. Bian, C. Zhang, R. Sankar, G. Chang, Z. Yuan, C.-C. Lee, S.-M. Huang, H. Zheng, J. Ma, D. S. Sanchez, B. Wang, A. Bansil, F. Chou, P. P. Shibayev, H. Lin, S. Jia, and M. Z. Hasan, Discovery of a Weyl fermion semimetal and topological Fermi arcs, Science 349, 613 (2015).

[12] J. Klotz, S.-C. Wu, C. Shekhar, Y. Sun, M. Schmidt, M. Nicklas, M. Baenitz, M. Uhlarz, J. Wosnitza, C. Felser, and B. Yan, Quantum oscillations and the Fermi surface topology of the Weyl semimetal NbP, Phys. Rev. B 93, 121105(R) (2016).

[13] F. Arnold, C. Shekhar, S.-C. Wu, Y. Sun, R. D. dos Reis, N. Kumar, M. Naumann, M. O. Ajeesh, M. Schmidt, A. G. Grushin et al., Negative magnetoresistance without well-defined chirality in the Weyl semimetal TaP, Nat. Commun. 7, 116152016.

[14] F. Arnold, M. Naumann, S.-C. Wu, Y. Sun, M. Schmidt, H. Borrmann, C. Felser, B. Yan, and E. Hassinger, Chiral Weyl Pockets and Fermi Surface Topology of the Weyl Semimetal TaAs, Phys. Rev. Lett. 117, 146401 (2016).

[15] X. Huang, L. Zhao, Y. Long, P. Wang, D. Chen, Z. Yang, H. Liang, M. Xue, H. Weng, Z. Fang et al., Observation of the Chiral-Anomaly-Induced Negative Magnetoresistance in 3D Weyl Semimetal TaAs, Phys. Rev. X 5, 031023 (2015).

[16] Z. Wang, Y. Zheng, Z. Shen, Y. Lu, H. Fang, F. Sheng, Y. Zhou, X. Yang, Y. Li, C. Feng, and Z.-A. Xu, Helicity-protected ultrahigh mobility Weyl fermions in NbP, Phys. Rev. B 93, 121112(R) (2016)

[17] C.-L. Zhang, S.-Y. Xu, I. Belopolski, Z. Yuan, Z. Lin, B. Tong, G. Bian, N. Alidoust, C.-C. Lee, S.-M. Huang, T.-R. Chang, G. Chang, C.-H. Hsu, H.-T. Jeng, M. Neupane, D. S. Sanchez, H. Zheng, J. Wang, H. Lin, C. Zhang et al., Signatures of the Adler-Bell-Jackiw chiral anomaly in a Weyl fermion semimetal, Nat. Commun. 7, 10735 (2016).

[18] Y. Li, Z. Wang, P. Li, X. Yang, Z. Shen, F. Sheng, X. Li, Y. Lu, Y. Zheng, and Z.-A. Xu, Negative magnetoresistance in Weyl semimetals $\mathrm{NbAs}$ and NbP: Intrinsic chiral anomaly and extrinsic effects, Front. Phys. 12, 127205 (2017).

[19] R. D. dos Reis, M. O. Ajeesh, N. Kumar, F. Arnold, C. Shekhar, M. Naumann, M. P. Schmidt, M. Nicklas, and E. Hassinger, Pressure tuning the Fermi surface topology of the Weyl semimetal NbP, New J. Phys. 18, 085006 (2016).

[20] K. Yoshida, A geometrical transport model for inhomogeneous current distribution in semimetals under high magnetic fields, J. Phys. Soc. Jpn. 40, 1027 (1976); An anomalous behavior of the longitudinal magnetoresistance in semimetals, 41, 574 (1976); Transport of spatially inhomogeneous current in a compensated metal under magnetic fields, III. A case of bismuth in longitudinal and transverse magnetic fields, J. Appl. Phys. 51, 4226 (1980).

[21] A. B. Pippard, Magnetoresistance in Metals (Cambridge University Press, Cambridge, UK, 1989).

[22] A. J. Kawabata, Theory of negative magnetoresistance, I. Application to heavily doped semiconductors, J. Phys. Soc. Jpn 49, 628 (1980).
[23] B. L. Altshuler and A. G. Aronov, in Electron-Electron Interaction in Disordered Solid, edited by A. L. Efros and M. Pollak (North-Holland, Amsterdam, 1985).

[24] D. V. Baxter, R. Richter, M. L. Trudeau, R. W. Cochrane, and J. O. Strom-Olsen, Fitting to magnetoresistance under weak localization in three dimensions, J. Phys. France 50, 1673 (1989).

[25] A. C. Hewson, The Kondo Problem to Heavy Fermions (Cambridge University Press, Cambridge, UK, 1993).

[26] J. Kondo, Resistance minimum in dilute magnetic alloys, Prog. Theor. Phys. 32, 37 (1964).

[27] X. Dai, Z. Z. Du, and H.-Z. Lu, Negative Magnetoresistance without Chiral Anomaly in Topological Insulators, Phys. Rev. Lett. 119, 166601 (2017).

[28] A. V. Andreev and B. Z. Spivak, Longitudinal Negative Magnetoresistance and Magnetotransport Phenomena in Conventional and Topological Conductors, Phys. Rev. Lett. 120, 026601 (2018).

[29] Y. Gao, S. A. Yang, and Q. Niu, Intrinsic relative magnetoconductivity of nonmagnetic metals, Phys. Rev. B 95, 165135 (2017).

[30] Y. Awashima and Y. Fuseya, Longitudinal and transverse magnetoresistance of $\mathrm{SrTiO}_{3}$ with a single closed Fermi surface, J. Phys.: Condens. Matter 31, 29LT01 (2019).

[31] A. C. Niemann, J. Gooth, S.-C. Wu, S. Bäßler, P. Sergelius, R. Hühne, B. Rellinghaus, C. Shekhar, V. Süß, M. Schmidt, C. Felser, B. Yan, and K. Nielsch, Chiral magnetoresistance in the Weyl semimetal NbP, Sci. Rep. 7, 43394 (2017).

[32] B. J. Ramshaw, K. A. Modic, A. Shekhter, Y. Zhang, E.-A Kim, P. J. W. Moll, M. D. Bachmann, M. K. Chan, J. B. Betts, F. Balakirev, A. Migliori, N. J. Ghimire, E. D. Bauer, F. Ronning, and R. D. McDonald, Quantum limit transport and destruction of the Weyl nodes in TaAs, Nat. Commun. 9, 2217 (2018).

[33] See Supplemental Material at http://link.aps.org/supplemental/ 10.1103/PhysRevMaterials.4.034201 for sample parameters, complete data, estimation of the paramagnetic impurity concentration, calculation of the orbital magnetoresistance using the DFT Fermi surfaces, and the complete fit results.

[34] H. K. Pal and D. L. Maslov, Necessary and sufficient condition for longitudinal magnetoresistance, Phys. Rev. B 81, 214438 (2010).

[35] C. Shekhar, A. K. Nayak, Y. Sun, M. Schmidt, M. Nicklas, I. Leermakers, U. Zeitler, Y. Skourskii, J. Wosnitza, Z. Liu, Y. Chen, W. Schnelle, H. Borrmann, Y. Grin, C. Felser, and B. Yan, Extremely large magnetoresistance and ultrahigh mobility in the topological Weyl semimetal candidate NbP, Nat. Phys. 11, 645 (2015).

[36] Y. Luo, N. J. Ghimire, E. D. Bauer, J. D. Thomson, and F. Ronning, "Hard" crystalline lattice in the Weyl semimetal NbAs, J. Phys.: Condens. Mat. 28, 055502 (2016).

[37] T. Besara, D. A. Rhodes, K.-W. Chen, S. Das, Q. R. Zhang, J. Sun, B. Zeng, Y. Xin, L. Balicas, R. E. Baumbach, E. Manousakis, D. J. Singh, and T. Siegrist, Coexistence of Weyl physics and planar defects in the semimetals TaP and TaAs, Phys. Rev. B 93, 245152 (2016).

[38] P. J. W. Moll, Focused ion beam microstructuring of quantum matter, Annu. Rev. Condens. Matter Phys. 9, 147 (2018).

[39] M. D. Bachmann, N. Nair, F. Flicker, R. Ilan, T. Meng, N. J. Ghimire, E. D. Bauer, F. Ronning, J. G. Analytis, and 
P. J. W. Moll, Inducing superconductivity in Weyl semimetal microstructures by selective ion sputtering, Sci. Adv. 3, e1602983 (2017).

[40] S. Hikami, A. I. Larkin, and Y. Nagaoka, Spin-orbit interaction and magnetoresistance in the two dimensional random system, Prog. Theor. Phys. 63, 707 (1980).
[41] H.-Z. Lu and S.-Q. Shen, Weak antilocalization and localization in disordered and interacting Weyl semimetals, Phys. Rev. B 92, 035203 (2015).

[42] M. Besser, Masters thesis, TU Dresden, 2018.

[43] A. B. Pippard, Longitudinal magnetoresistance, Proc. R. Soc. London, Ser. A 282, 464 (1964). 